\title{
A revision of the Japanese genera and species of the subfamily Hadrotarsinae (Araneae: Theridiidae)
}

\author{
Hajime Yoshida \\ 7-16, Kagota 2 Chome, Yamagata-shi, Yamagata, 990-2484 Japan \\ E-mail: araneae@mb.infoweb.ne.jp
}

\begin{abstract}
Japanese genera and species of the subfamily Hadrotarsinae Thorell 1881 (Araneae: Theridiidae) are revised. The presence of specialized ventral setae of first tarsi, two pairs of seminal receptacles and a dorso-ventrally flattened craw of female palpus characterize Hadrotarsinae. A key to the genera of Hadrotarsinae is given.

Nineteen species belonging to six genera of Hadrotarsinae are recorded from Japan. A new genus, Yaginumena, is described. Two genera, Trigonobothrys Simon 1889 and Emertonella Bryant 1949, are resurrected, and a genus, Lasaeola Simon 1881, is recorded from Japan for the first time. Twelve species are newly transferred from Dipoena Thorell 1869 to those genera: Yaginumena castrata (Bösenberg \& Strand 1906), Y. mutilata (Bösenberg \& Strand 1906), Y. maculosa (Yoshida \& Ono 2000), Lasaeloa okinawana (Yoshida \& Ono 2000), L. yoshidai (Ono 1991), L. yona (Yoshida \& Ono 2000), Trigonobothrys japonicus (Yoshida 1985), T. amamiensis (Yoshida 1985), T. martinae (Roberts 1983), T. flavomarginatus (Bösenberg \& Strand 1906), T. mustelinus (Simon 1889) and T. nigromaculatus (Yoshida 1987); and a species from Euryopis Menge 1868: Emertonella taczanowskii (Keyserling 1886). A new species, Dipoena nipponica, is described from Iriomote Is., the Nansei Islands. A species, Dipoena immaculata Zhu 1998 described from China, is newly synonymized with T. flavomarginatus, and a genus, Pselothorax Chamberlin 1948, with Lasaeola.
\end{abstract}

Key words - Hadrodarsinae, Theridiidae, Japan, Yaginumena, Trigonobothrys, Emertonella, revision.

\section{Introduction}

Thorell (1881) had established the spider family Hadrotarsidae, but later Wunderlich (1978) synonymized Hadrotarsidae with Theridiidae. Forster et al. (1990) proposed the subfamily Hadrotarsinae for 11 genera (Dipoena Thorell 1869, Euryopis Menge 1868, Anatea Berland 1927, Audifia Keyserling 1884, Dipoenata Wunderlich 1988, Euryopena Wunderlich 1992, Gmogola Keyserling 1889, Guaraniella Baert 1984, Hadrotarsus Thorell 1881, Lasaeola Simon 1881 and Yoroa Baert 1984) on the basis of the presence of specialized ventral setae of first tarsi, two pairs of seminal receptacles, a dorso-ventrally flattened craw of female palpus and a series of ridged projections on anterior spinnerets. All the members of Hadrotarsinae may be specialized as predators of ants.

Wunderlich (1988) resurrected the genus Lasaeola that had been synonymized with Dipoena. Lasaeola prona (Menge 1868), which occurs also in Japan (Yoshida \& Ono 2000 ) is the type species of this genus. In this paper, I resurrect two genera, Trigonobothrys Simon 1889 and Emertonella Bryant 1949, that have been synonymized each with Dipoena and Euryopis. In addition to them, a new genus, Yaginumena, is described. Thus, the subfamily Hadrotarsinae consists of a total of 14 genera.
In Japan, only two genera, Dipoena and Euryopis, have been used under the subfamily Hadrotarsinae (Tanikawa 2000). Fourteen species belonging to four species groups (sulfurica, prona, lineatipes and nigra-groups) have been recorded from Japan in the genus Dipoena s. lat. (Yoshida $\&$ Ono 2000). In this paper, I treated one species of the sulfurica group as the genus Dipoena s. str., four of the prona (hamata) group as Lasaeola, six of the lineatipes group as Trigonobothrys, and three of the nigra group as Yaginumena new genus. In addition to them, a new species of Dipoena is described from Iriomote Is., the Nansei Islands. On the other hand, in the genus Euryopis s. lat. five species are known from Japan (Yaginuma 1986; Yoshida $1992,1997,2000)$. One of them is newly transferred to Emertonella and three of them belong to Euryopis s. str. Remaining one, Euryopis iharai Yoshida 1992, is excluded in this paper, because systematic position of it is uncertain. In summary, a total of 19 species belonging to six genera are recorded from Japan under the subfamily Hadrotarsinae.

Specimens used in this paper are the same as those of "additional record(s)" and "specimens examined" listed by Yoshida \& Ono (2000) and Yoshida (1992, 1997, 2000). Prefectural names are capitalized in the list of "additional record(s)". Depositories of specimens excluding my private collection are given in abbreviations: ASJ, Arachnological 
Society of Japan, Otemon Gakuin University, Osaka; BMNH, Museum of Natural History, London, England; CMM, private collection of Mayumi Matsuda, Hokkaido; HEC, Hebei Educational College, Shijiazhuang, China; HUW, Laboratory of Pest Natural Enemies, Department of Biology, Hubei University, Wuhan, China; IRRI, International Rice Research Institution, Manila, the Philippines; KUT, Kyungpook University, Taegu, Korea; MCZ, Museum of Comparative Zoology, Massachusetts, USA; NHMS, Natural History Museum, Stockholm, Sweden; NSMT, National Science Museum, Tokyo; OMNH, Osaka Museum of Natural History, Osaka; PAS, Polish Academy of Science, Warsaw, Poland; SMF, Senckenberg Museum Frankfurt am Main, Germany.

\section{Taxonomy}

Subfamily Hadrotarsinae Thorell 1881

[Japanese name: Mijingumo aka]

Hadrotarsidae Thorell 1881, p. 190 (established as a family); Simon 1893, p. 305; Petrunkevitch 1928, p. 36; Hickman 1942, p. 148; Baert 1984 a, p. 608; Baert 1984 b, p. 229.

Hadrotarsinae: Forster et al. 1990, p. 111.

Euryopeae Simon 1894, p. 524 (established as a genus group); Wiehle 1937, p. 131.

Dipoeneae Simon 1894, p. 564 (established as a genus group); Wiehle 1937, p. 180.
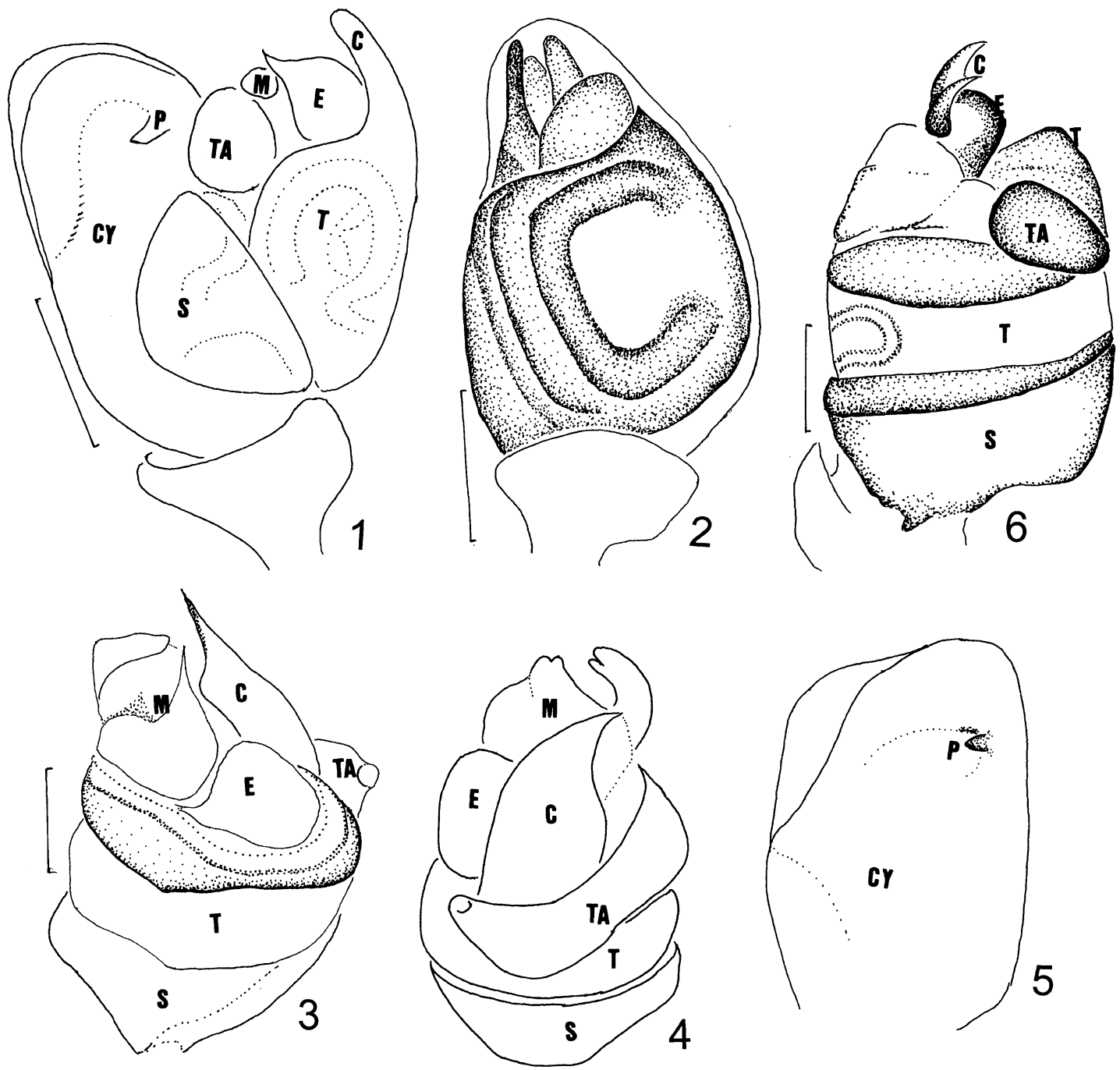

Figs. 1-6. Yaginumena mutilata (Bösenberg \& Strand 1906), 合 from Higashine-shi, Yamagata Pref. (1), Y. maculosa (Yoshida \& Ono 2000), 令 from Nagoya-shi, Aichi Pref. (2), Lasaeola prona (Menge 1868), 令 from Osaka-shi, Osaka Pref. (3-5) and Trigonobothrys amamiensis (Yoshida 1985), $\hat{\delta}$ from Shingu-mura, Ehime Pref. (6), left palpus - 1, expanded, prolateral view; 2, ventral view; 3-4, 6, bulb expanded (cymbium excluded), prolateral (3), retrolateral (4) and dorsal (6) view; 5, cymbium, ventral view. Scales: $0.1 \mathrm{~mm}$. Abbreviations: C, conductor; E, embolus; M, median apophysis; T, tegulum; TA, tegular apophysis; $\mathrm{S}$, subtegulum; CY, cymbium; $\mathrm{P}$, paracymbium. 
Diagnosis. This subfamily is easily distinguished from the other subfamilies of Theridiidae by having specialized ventral setae of first tarsi, two pairs of seminal receptacles and a dorso-ventrally flattened craw of female palpus.

Notes. Fourteen genera of the family Theridiidae belong to this subfamily. Six of them, Dipoena Thorell 1869, Yaginumena new genus, Lasaeola Simon 1881, Trigonobothrys Simon 1889, Euryopis Menge 1868 and Emertonella Bryant 1949, are confirmed in Japan. This subfamily can be treated as a family, but I follow Forster et al. (1990) in this paper.

Type genus. Hadrotarsus Thorell 1881.

Key to the Japanese genera of the subfamily Hadrotarsinae

1. Colulus small with two setae; male carapace high, with

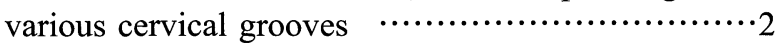

- Colulus absent; male carapace not so high, without dis-

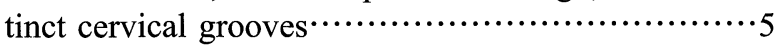

2. Abdomen with distinct black and white flecks …..... Dipoena Thorell 1869

- Abdomen without distinct black and white flecks $\cdots 3$

3. Male carapace with high head region, without large cervical grooves; conductor conjugated with tegulum

- Yaginumena new genus

- Male carapace cylindrical and high, with various cervical grooves; conductor separated from tegulum $\cdots \cdots \cdot 4$

4. Cylinder of male carapace not so large; tegular apophysis, conductor and embolus larger and relatively complex; median apophysis present

- Lasaeola Simon 1881

- Cylinder of male carapace large and high; tegular apophysis, conductor and embolus small and simple; median apophysis absent ...................................

-Trigonobothrys Simon 1889

5. Carapace with high head region; abdomen blackish brown with large silver flecks; cymbium of male palpus without projection ‥Emertonella Bryant 1949

- Carapace without high head region; abdomen blackish brown to black with white or yellow flecks; cymbium of male palpus with a distal projection

-Euryopis Menge 1868

Dipoena Thorell 1869 s. str.

[Japanese name: Mijingumo zoku]

Dipoena Thorell 1869, p. 91.

Dipoena s. lat.: Simon 1894, p. 567; Wiehle 1937, p. 180; Saito 1941, p. 160; Levi 1953, p. 3; Yaginuma 1960, p. 39; Levi \& Levi 1962, p. 40; Levi 1963, p. 136; Yaginuma 1968, p. 34; Yaginuma 1986, p. 42; Wunderlich 1987, p. 210; Zhu 1998, p. 224; Yoshida \& Ono 2000, p. 127.

Diagnosis. This genus resembles related genera of the subfamily Hadrotarsinae, but is distinguished from Yaginumena by having small tegulum of male palpus, and black and white coloration of abdomen, and from Lasaeola and Trigonobothrys by having non-cylindrical carapace without various thoracic groove in males. This genus is easily distinguished from Euryopis and Emertonella by the presence of colulus with two setae. Only the species of $D$. sulfurica group belong to this genus.

Description. Superficial appearance is similar to that of other genera of Hadrotarsinae. Carapace oval, head region high (Figs. 8, 15). Abdomen oval with distinct black and white flecks (Figs. 7, 14). Two seminal receptacles connecting with sclerotized duct (Fig. 11). Male palpus with free median apophysis (Figs. 12-13, 18-20).

Type species. Atea melanogaster C. L. Koch 1837

\section{Dipoena nipponica new species}

[Japanese name: Tanikawa-mijingumo]

(Figs. 7-13)

Diagnosis. This species is easily distinguished from the other species of the genus Dipoena by having 1) thin, long and anticlockwise embolus of male palpus (left), 2) thick and long conductor of male palpus, and 3 ) the duct of female internal genitalia forming a dorsally thick circle.

Description. Carapace oval, head region projecting; fovea longitudinal (Figs. 7-8). Abdomen globular. Female palpal claw dorso-ventrally flattened (Fig. 9). Anterior median eyes largest.

Female genitalia as shown in Figs. 10-11: epigynum with an antero-median projection; duct thick and long, forming a dorsally large circle; seminal receptacles two pairs.

Male palpus as shown in Figs. 12-13: large concavity present between conductor and tegular apophysis; embolus thin and long, anticlockwise (left palpus); base of embolus with median apophysis; conductor thick and long; tegular apophysis projecting distally.

Coloration. Carapace brown, marginally with dusky wide flecks. Legs and palpi brown with black flecks. Chelicerae, maxillae and labium grayish brown. Abdomen brown with many distinct black flecks and white pigments; venter with large black flecks between spinnerets and epigynum, and anterior part of epigastric furrow.

Measurements (in mm. ㅇ allotype/s holotype). Body length 1.92/1.71. Carapace length $0.76 / 0.82$; width $0.71 / 0.71$; height 0.76/0.79. Abdomen length 1.13/0.89; width 1.26/1.03; height $1.18 / 0.92$. First leg: femur $0.74 / 0.79$; patella and tibia $0.76 / 0.87$; metatarsus $0.45 / 0.50$; tarsus $0.32 / 0.34$. Second patella and tibia $0.66 / 0.74$; third patella and tibia 0.55/0.53; fourth patella and tibia $0.71 / 0.71$. Diameters: anterior median eye $0.12 / 0.12$; anterior lateral eye $0.05 / 0.05$; posterior median eye $0.09 / 0.08$; posterior lateral eye $0.08 / 0.08$. Distances: between anterior median eyes $0.07 / 0.08$; between anterior median and lateral eyes $0.03 / 0.03$; between posterior median eyes $0.04 / 0.05$; between posterior median and lateral eyes 0.08/0.08. Median ocular area, anterior width $0.28 / 0.29$; posterior width $0.22 / 0.22$; length $0.22 / 0.22$. 

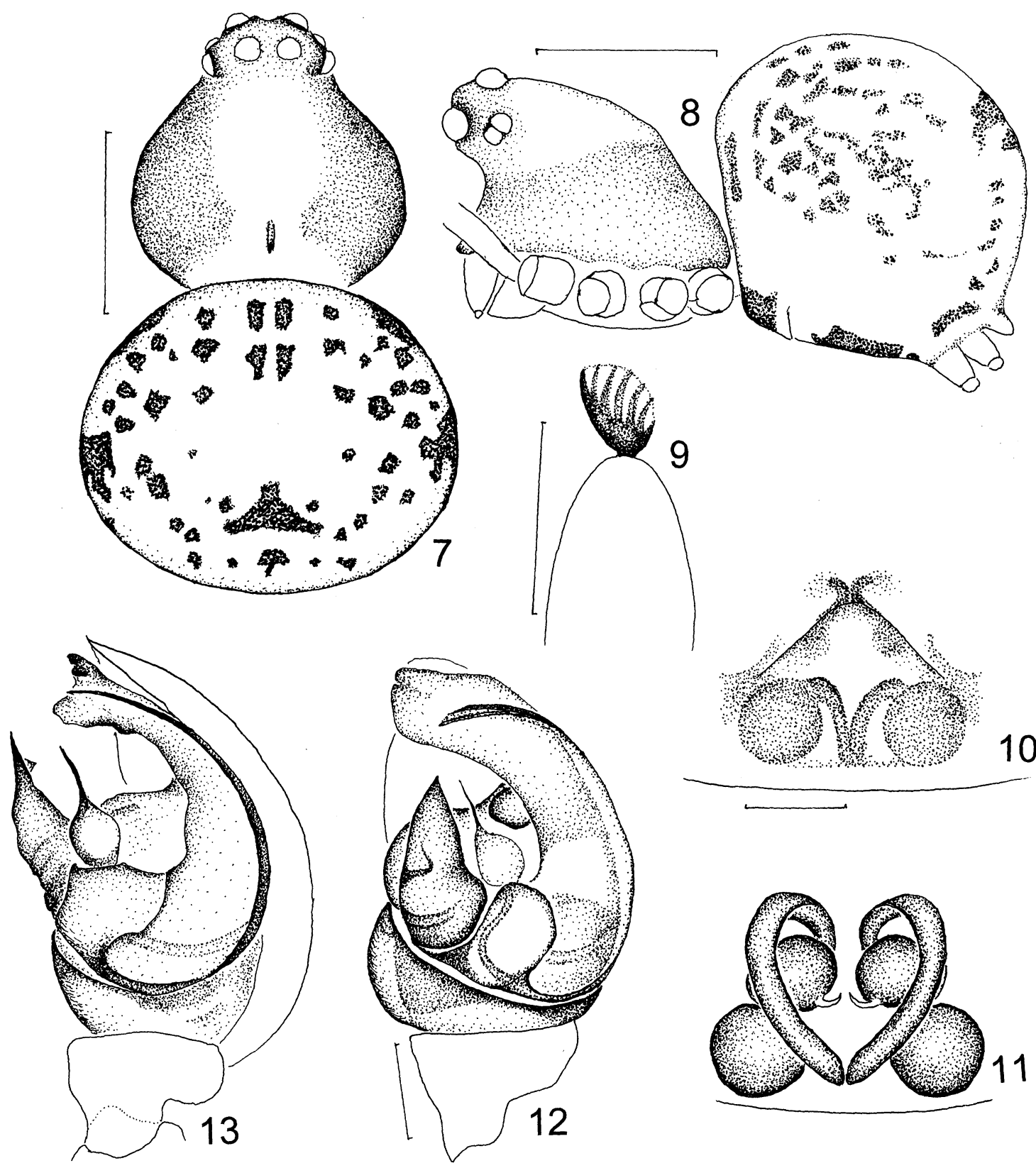

Figs. 7-13. Dipoena nipponica new species, o allotype and $\hat{o}$ holotype -7 , male carapace and abdomen, dorsal view; 8 , male, of lateral view; 9, craw of female left palpus, dorsal vies: $0.5 \mathrm{~mm}(7-8)$ and $0.1 \mathrm{~mm}(9-13)$.

Variation. Body length, $1.8-1.9 \mathrm{~mm}$ in female, $1.6-1.7$ $\mathrm{mm}$ in male.

Type series. Holotype: $\hat{\delta}$, Sonai, 11-VIII-1985 (NSMT-Ar 190). Allotype: ㅇ, Komi, 6-VIII-1987, (NSMT-Ar 5191). Paratypes: 1, 5-I-1992, 1ㅇ, 14-VIII-1992, Sonai, (NSMT-Ar 5192-5193); 1+9, Shirahama, 25-VIII-1988, (NSMT-Ar 5194); 1 ई. Funaura, 3-I-1986, (NSMT-Ar 5195). All collected by A. Tanikawa on Iriomote Is., Okinawa Pref., Japan.

Distribution. Japan: Iriomote Is. of the Yaeyama Islands (southernmost islands of the Nansei Islands).
Dipoena punctisparsa Yaginuma 1967 [Japanese name: Shimofuri-mijingumo]

(Figs. 14-20)

Dipoena punctisparsa Yaginuma 1967, p. 97, fig. 3f-h (holotype: of from Niijima Island, the Izu Islands, Tokyo Prefecture, Japan, 2-VI-1967, M. Ohno leg., in ASJ; examined)-Yaginuma 1968, p. 129, text-fig. 107; Yaginuma 1986, p. 42, text-fig. 23-4; Chikuni 1989, p. 36, fig. 31; Yoshida \& Ono 2000, p. 149. 

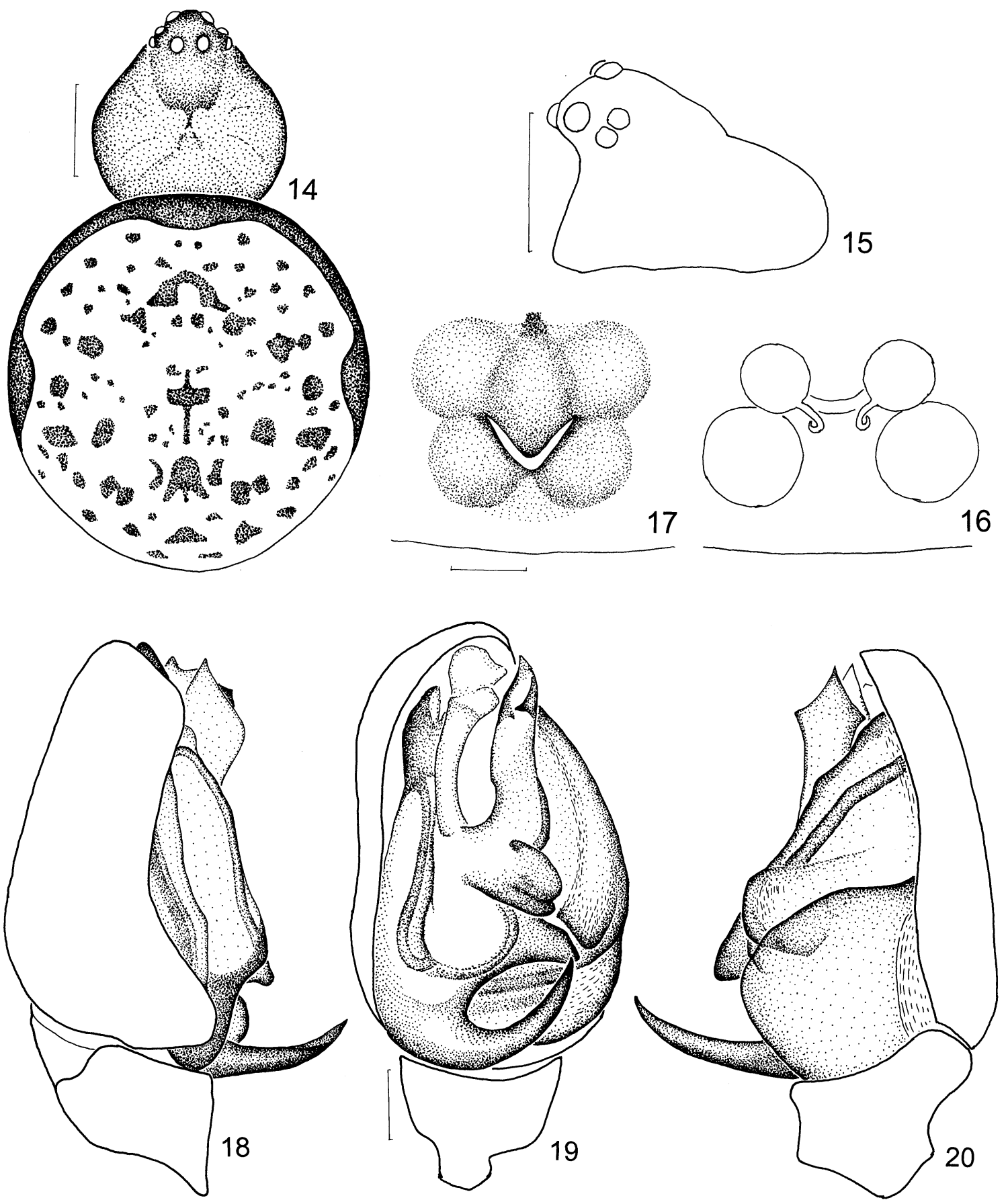

Figs. 14-20. Dipoena punctisparsa Yaginuma 1967, 우송 from Yokosuka-shi, Kanagawa Pref.-14, female carapace and abdomen, dorsal view; 15 , male carapace, lateral view; 16, epigynum, ventral view; 17 , female internal genitalia, dorsal view; $18^{-}$ 20, male left palpus, prolateral (18), ventral (19) and retrolateral (20) view. Scales: $0.5 \mathrm{~mm}(14-15)$ and $0.1 \mathrm{~mm}\left(16^{-20)}\right.$.

Additional record. HOKKAIDO: 2우, Mt. Esashigyu, Kamikawa-cho, 1-VIII-1984, N. Yasuda leg.

Distribution. Japan: Hokkaido, Honshu and Kyushu. Korea.

Notes. Epigynum with V-shaped depression (Fig. 16); seminal receptacles two pairs and circular (Fig. 17). Tegulum of male palpus ventrally with a large projection
(Figs. 18-20).

\section{Yaginumena new genus}

[Japanese name: Yaginuma-mijingumo zoku]

Diagnosis. This genus resembles Dipoena, but is distinguished from the latter by having dark abdomen, large 

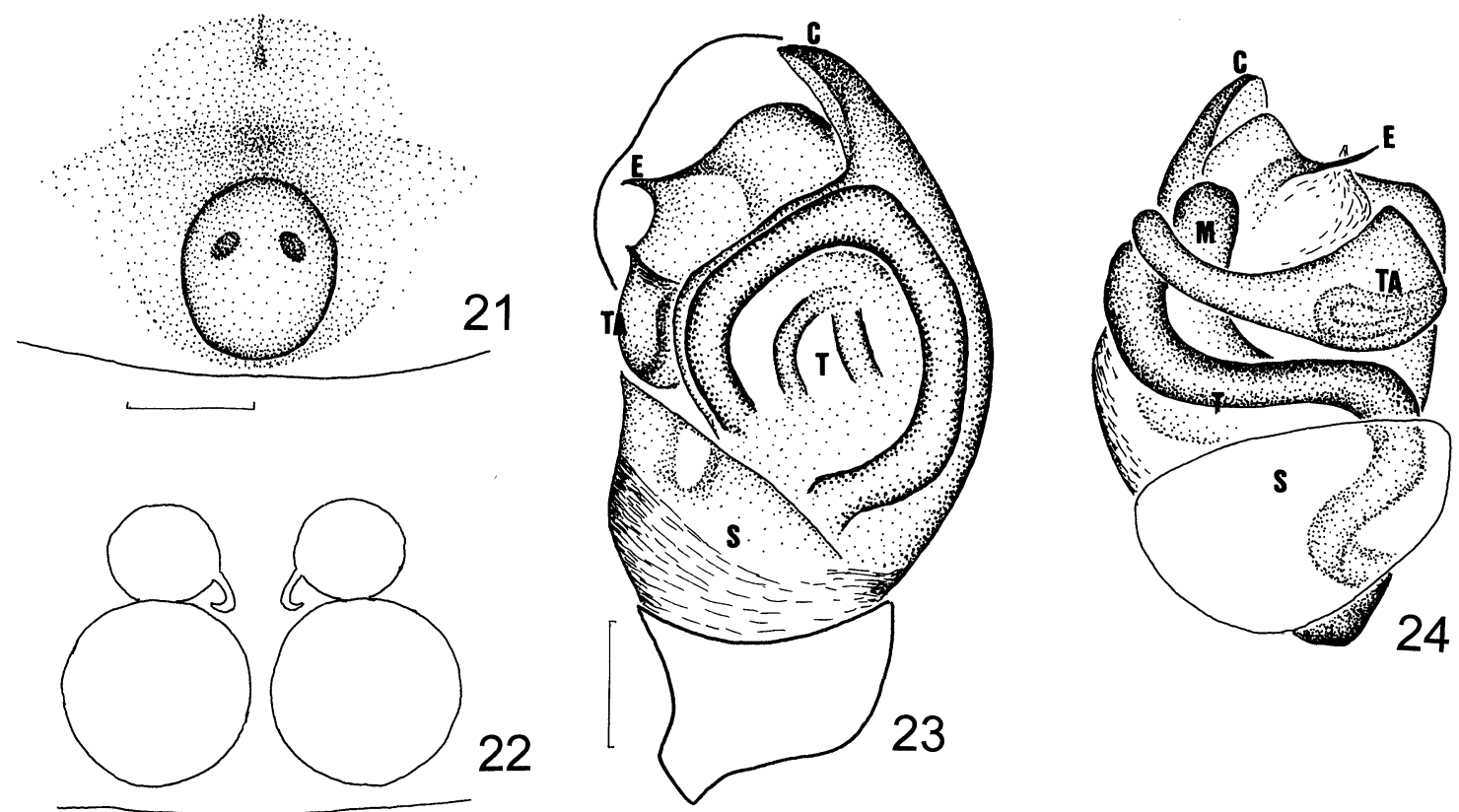

Figs. 21-24. Yaginumena castrata (Bösenberg \& Strand 1906), 우 f from Mogami-machi, Yamagata Pref.-21, epigynum, ventral view; 22, female internal genitalia, dorsal view; 23-24, male left palpus, ventral (23) and dorsal (24, expanded and cymbium excluded) view. Scales: $0.1 \mathrm{~mm}$. Abbreviations: C, conductor; E, embolus; M, median apophysis; T, tegulum; TA, tegular apophysis; S, subtegulum.

tegulum and small embolus of male palpus, and large sclerotized plate of epigynum.

Description. Carapace oval with high head region without distinct fovea. Abdomen oval and usually dark color without distinct light flecks. Epigynum with a sclerotized oval plate that has two openings (Figs. 21-22). Tegulum of male palpus large with a thick and long internal duct; tegular apophysis, median apophysis, embolus and conductor small; conductor conjugated with tegulum (Figs. 1-2, 23-24).

Notes. All the species of the D. nigra group designated by Levi (1953) belong to this genus.

Etymology. The generic name is dedicated to the late Dr. Takeo Yaginuma, Osaka, and is a feminine in gender.

Type species. Dipoena castrata Bösenberg \& Strand 1906.

\section{Yaginumena castrata (Bösenberg \& Strand 1906) new combination}

[Japanese name: Bokashi-mijingumo]

(Figs. 21-24)

Dipoena castrata Bösenberg \& Strand 1906, p. 149, pl. 5, fig. 50, pl. 12, figs. 246-247 (syntypes: 1 우 and 4 juveniles from Saga, Japan, the middle of June, W. Dönitz leg., SMF 3069; not examined)-Saito 1941, p. 161, fig. 182; Yaginuma 1960, p. 39, pl. 11, fig. 67, text-fig. 39; Yaginuma 1968, p. 39, pl. 11, fig. 67, text-fig. 39; Yaginuma 1986, p. 42, pl. 10, fig. 1 , text-fig. 23-1; Chikuni 1989, p. 36, fig. 30; Zhu 1998, p. 248, fig. 164; Song et al. 1999, p. 110, fig. 53 I-J, N-O; Yoshida
\& Ono 2000, p. 143, figs. 31-32.

Dipoena uniforma Bösenberg \& Strand 1906, p. 151, pl. 12, fig. 285 (syntypes: $1 \hat{\delta}$ and 1 juvenile from Kompira, Saga, Japan, W. Dönitz leg., SMF 3075; not examined)-Saito 1941, p. 163, fig. 186.

Distribution. Japan: Hokkaido, Honshu, Shikoku and Kyushu. Korea and China.

\section{Yaginumena mutilata (Bösenberg \& Strand 1906) new combination}

[Japanese name: Ko-akakuro-mijingumo]

(Fig. 1)

Dipoena mutilata Bösenberg \& Strand 1906, p. 150, pl. 12, fig. 275 (holotype: 우 from Japan, W. Dönitz leg., SMF 3073; examined) - Saito 1941, p. 163, fig. 185; Yoshida \& Ono 2000 , p. 139 , figs. $21-30$.

Dipoena longisternum Bösenberg \& Strand 1906, p. 152, pl. 10, fig. 189 (holotype: ㅇ from Kompira, Saga, Japan, W. Dönitz leg., SMF 3005; examined)-Saito 1941, p. 162, fig. 184.

Additional record. HOKKAIDO: 1, Okushiri Is., 28-VII1964, M. Ohno leg. (OMNH).

Distribution. Japan: Hokkaido, Honshu and Kyushu. Korea. 


\section{Yaginumena maculosa (Yoshida \& Ono 2000) new combination \\ [Japanese name: Madara-mijingumo]}

(Fig. 2)

Dipoena maculosa Yoshida \& Ono 2000, p. 147, figs. 37-39 (holotype: $\hat{\delta}$ from Numa, Tamano-shi, Okayama Prefecture, Japan, 1-VII-1997, K. Nojima leg., NSMT-Ar 4506; examined).

Additional record. AICHI: $1 \hat{\jmath}$, Otaka-ryokuchi Park, Midoriku, Nagoya-shi, 27-V-2001, K. Ogata leg.

Distribution. Japan: Honshu (Aichi and Okayama Prefectures).

Notes. Male palpus of the holotype is expanded (Yoshida \& Ono 2000, figs. 38-39), but that of a specimen collected from Nagoya-shi, Aichi Pref., is not expanded (Fig. 2).

\section{Lasaeola Simon 1881}

[Japanese name: Aichi-mijingumo zoku]

Pachydactylus Menge 1868, p. 176 (homonym of Pachydactylus Wiegmann 1834).

Lasaeola Simon 1881, p. 136; Wunderlich 1988, p. 148.

Pselothorax Chamberlin 1948, p. 541. New Synonymy

Diagnosis. This genus is similar to Dipoena, Yaginumena and Trigonobothrys in general appearance, but is distinguished from Dipoena by high male carapace and dusky coloration of abdomen, from Yaginumena by high male carapace and large conductor and embolus, and from Trigonobothrys by the presence of median apophysis, and complicated and projecting tegular apophysis of male palpus.

Description. Carapace oval with head region high, sometimes cylindrical and specialized in male. Eyes almost equal. Tegular apophysis of male palpus complicated and projecting; tegulum relatively small; embolus and conductor large; paracymbium hooked (Figs. 3-5). Epigynum with a distinct depression.

Notes. This genus had been synonymized with Dipoena, but Wunderlich (1988) resurrected it as an independent genus. Ivie (1967) synonymized Pselothorax Chamberlin 1948 (type species from North America) with Dipoena, but I newly synonymize it with this genus. All the species of the D. prona group designated by Levi (1953) belong to this genus.

Type species. Pachydactylus ponus Menge 1868.

Lasaeola prona (Menge 1868)

[Japanese name: Aichi-mijingumo]

(Figs. 3-5)

Pachydactylus ponus Menge 1868, p. 177 (holotype: 우 from Weichselmünde forest near Gdańsk, Poland; not examined).
Lasaeola prona: Simon 1881, p. 145.

Dipoena prona: Wiehle 1937, p. 186, figs. 184-185; Levi 1963, p. 145; Roberts 1985, p. 176, pl. 105 b, text-fig. 78b; Yoshida \& Ono 2000 , p. 149 , figs. $40-47$.

Dipoena hamata Tullgren 1949, p. 50, figs. 10-11 (holotype: $\hat{\delta}$ from St. Kalsö, Sweden, in NHMS; not examined)-Levi 1953 , p. 30 , figs. 50-59, 105-106.

Additional record. OSAKA: 2우소, riverside of Yodogawa, Toyosato, Higashi-yodogawa-ku, Osaka-shi, 13-VII-2000, K. Nojima leg.

Distribution. Japan: Honshu (Aichi and Osaka Prefectures). Europe and North America.

Notes. Though Theridion triste Hahn 1833 is recorded as the type species of this genus by Platnick (2001), this species is the type species of Pachydactylus Menge 1868.

\section{Lasaeloa okinawana (Yoshida \& Ono 2000) new combination}

[Japanese name: Okinawa-mijingumo]

Dipoena amamiensis (in part): Yoshida 1991, p. 35, figs. 5-6; Zhu 1998, p. 242, fig. 159-B, E.

Dipoena okinawana Yoshida \& Ono 2000, p. 146, figs. 33-36 (holotype: 令 from Mt. Banna-dake, Ishigaki-jima Island, Okinawa Prefecture, Japan, 11-VII-1976, H. Yoshida leg., NSMT-Ar 4504; examined).

Distribution. Japan: Nansei Islands (Okinawa and Ishigaki Islands).

Notes. Yoshida \& Ono (2000) made this species belong to the $D$. nigra group (in this paper, the genus Yaginumena). However, I consider this species should be treated under Lasaeloa on the basis of the characteristics of male palpus.

\section{Lasaeola yoshidai (Ono 1991) new combination [Japanese name: Yoshida-mijingumo]}

Dipoena yoshidai Ono, in Ono et al. 1991, p. 91, figs. 2-8 (holotype: $\uparrow$ from Koetoi, $20 \mathrm{~m}$ alt., Wakkanai-shi, Hokkaido, Japan, 10-VII-1990, H. Ono leg., NSMT-Ar 2098; examined)Yoshida \& Ono 2000, p. 152, figs. 48-53.

Distribution. Japan: Hokkaido and Honshu.

\section{Lasaeola yona (Yoshida \& Ono 2000) new combination}

[Japanese name: Yona-mijingumo]

Dipoena yona Yoshida \& Ono 2000, p. 153, figs. 54-56 (holotype: 只 from Experiment Forest of the University of the Ryukyus, Yona, Okinawa-jima Island, Okinawa Prefecture, Japan, 28-III-1997, A. Tanikawa leg., NSMT-Ar 4509; examined).

Distribution. Japan: Nansei Islands (Okinawa Is.). 


\section{Trigonobothrys Simon 1889 \\ [Japanese name: Tsutsu-mijingumo zoku]}

Trigonobothrys Simon 1889a, p. 230.

Diagnosis. This genus resembles Lasaeola, but is distinguishable from it by having male palpus with small embolus and conductor and without median apophysis, and epigynum with a small scapus.

Description. Carapace oval with high head region in female, wholly high and cylindrical with a wide and deep cervical groove (Fig. 25). Abdomen usually sclerotized in both sexes, with a dorsal scutum in male. Epigynum with a small scapus (Figs. 26-27). Embolus and conductor of male palpus small; median apophysis absent (Figs. 6, 28).

Notes. This genus has been synonymized with Dipoena, but I resurrect it as a distinct genus. All the species of the $D$. lineatipes group designated by Levi (1953) belong to this genus.

Type species. Trigonobothrys excisus Simon 1889.

\section{Trigonobothrys japonicus (Yoshida 1985) new combination \\ [Japanese name: Yamato-mijingumo]}

Pholcomma japonicum Yoshida 1985, p. 10, figs. 3, 8-11 (holotype: + + from Keishin-no-taki, the foot of Mt. Nishikoma-dake, Nagano Prefecture, Japan, 9-VIII-1974, Y. Chikuni leg., in ASJ; examined)-Chikuni 1989, p. 31, fig. 9. Dipoena japonica (in part): Yoshida 1991, p. 33.

Dipoena japonica: Yoshida \& Ono 2000, p. 128, figs. 1-6.

Dipoena kayaensis Paik 1996, p. 42, figs. 1-14 (holotype: $\hat{o}$ from Mt. Kayasan, Hein-sa, Korea, 10-VII-1974, K. Y. Paik leg., in KUT; not examined).

Additional record. MIE: 1\}, Kiho-cho, 22-V-1971, K. Kaihotsu leg. (OMNH).

Distribution. Japan: Honshu (Nagano, Aichi, Mie and Okayama Prefectures). Korea.

\section{Trigonobothrys amamiensis (Yoshida 1985) new combination \\ [Japanese name: Amami-mijingumo]}

(Fig. 6)

Pholcomma amamiense Yoshida 1985, p. 11, figs. 4-5, 11-12 (holotype: 우 from Naze, Amami-oshima Island, Kagoshima Prefecture, Japan, 27-III-1978, H. Yoshida leg., in ASJ; examined).

Dipoena japonica (in part): Yoshida 1991, p. 33, figs. 1, 3-4.

Dipoena amamiensis (in part): Yoshida 1991, p. 35.

Dipoena amamiensis: Paik 1995 b, p. 33, figs. 7-12; Yoshida \& Ono 2000, p. 130, figs. 7-10.

Distribution. Japan: Honshu (Tokyo, Shizuoka, Aichi,
Osaka, Okayama and Hiroshima Prefectures), Shikoku (Ehime Pref.) and the Nansei Islands (Amami-oshima Is.). Korea and China.

\section{Trigonobothrys martinae (Roberts 1983) new combination \\ [Japanese name: Hoshi-mijingumo]}

Dipoena martinae Roberts 1983, p. 227, figs. 32-35 (holotype: o from Grande Terre, Wilson's Well, Aldabra, western Indian Ocean, 9-III-1974, in BMNH; not examined)-Zhu 1998, p. 236, fig. 154; Song et al. 1999, p. 112, fig. 55C-D, K-L; Yoshida \& Ono 2000, p. 132, figs. 11-16.

Dipoena japonica (in part): Yoshida 1991, p. 10, fig. 2.

Dipoena decamaculata Chen et al. 1992, p. 270, figs. 1-5 (holotype: 令 from Xiushui County, Jiangxi Province, China, 10-IX-1991, in HUW; not examined).

Dipoena coreana Paik 1995b, p. 32, figs. 1-6 (holotype: $\hat{\delta}$ from Jinjoo, Kyungnam, Korea, 13-VIII-1981, B. K. Seo leg, in KUT; not examined).

Dipoena ruedai Barrion \& Litsinger 1995, p. 454, fig. 274 (holotype: $\hat{\delta}$ from Magasaysay Village, Siniloan, Laguna Province, Luzon Island, Philippines, 12-XII-1984, A.T. Barrion leg., in IRRI; not examined).

Distribution. Japan: Nansei Islands (Iriomote Is.). Korea, China, the Philippines and the Aldabra Islands (West Indian Sea).

\section{Trigonobothrys flavomarginatus \\ (Bösenberg \& Strand 1906) new combination \\ [Japanese name: Kiberi-mijingumo]}

(Figs. 25-28)

Dipoena flavomarginata Bösenberg \& Strand 1906 p. 151, pl. 12, fig. 297 (syntypes: 웅 from Saga, Japan, in SMF; not examined)-Saito 1941, p. 162, fig. 183; Ohno \& Yaginuma 1968 , p. 27, fig. 2; Yaginuma 1986, p. 42, pl. 10, fig. 23-2, text-fig. 23-2; Chikuni 1989, p. 36, fig. 33; Zhu 1998, p. 231, fig. 150; Song et al. 1999, p. 110, fig. 53K-L, P; Yoshida \& Ono 2000 , p. 136 , figs. $18-20$.

Dipoena immaculata Zhu 1998, p. 251, fig. 167 (holotype: $\hat{\delta}$ from Xuanen County, Hubei Province, China, 26-V-1989, M. Zhu leg., in HEC; not examined). New synonymy.

Distribution. Japan: Honshu, Shikoku and Kyushu. China.

Notes. I newly synonymize Dipoena immaculata Zhu 1998 described from China with T. flavomarginatus (Böse nberg \& Strand 1906) described from Japan, because figures of $D$. immaculata coincide with those of $T$. flavomarginatus (Figs. 25-28).

\section{Trigonobothrys mustelinus (Simon 1889)} new combination

[Japanese name: Kani-mijingumo] 

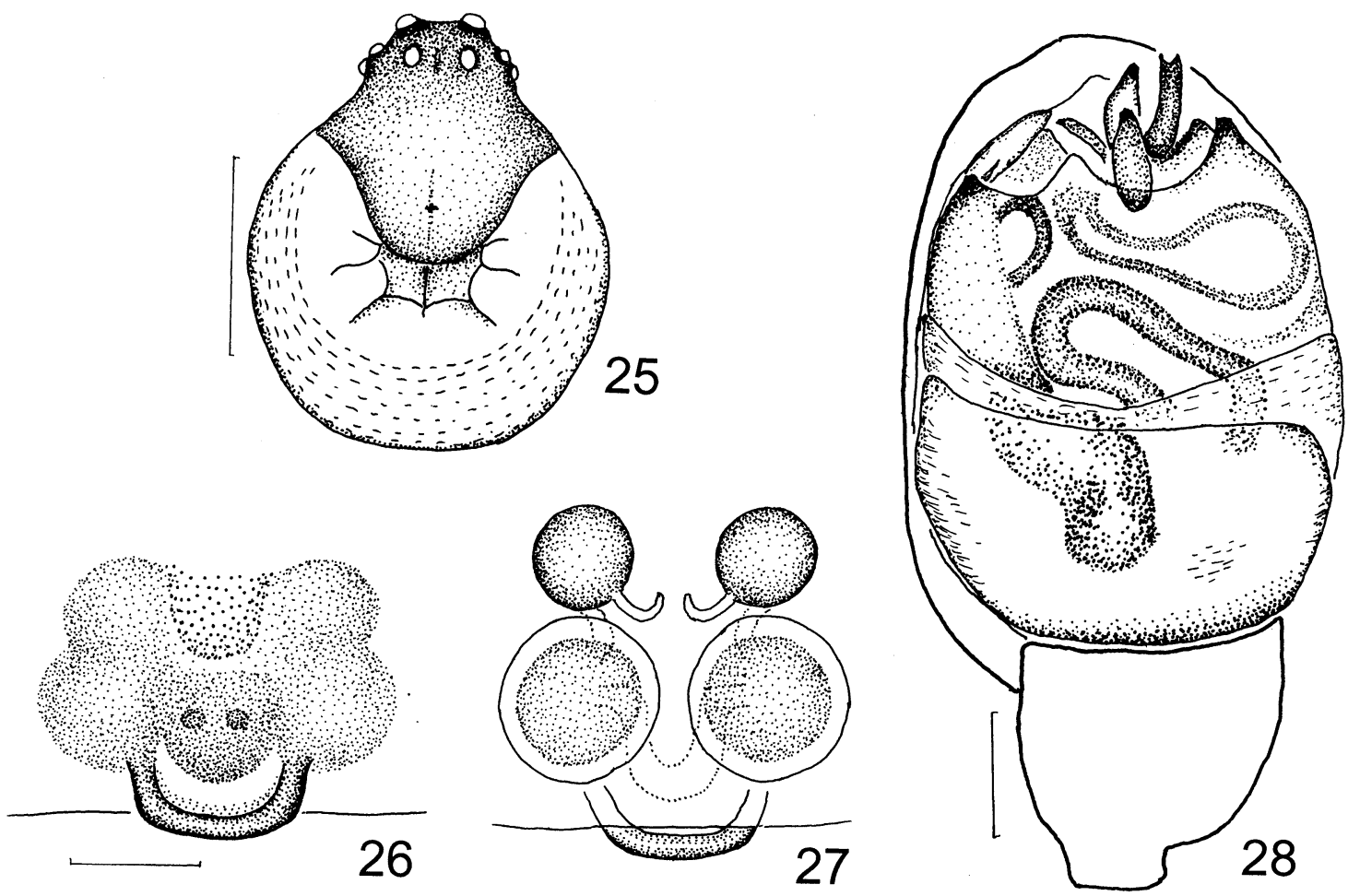

Figs. 25-28. Trigonobothrys flavomarginatus (Bösenberg \& Strand 1906), 우 f from Yamagata-shi, Yamagata Pref. 一25, male carapace, dorsal view; 26, epigynum, ventral view; 27 , female internal genitalia, dorsal view; 28 , male left palpus, ventral view. Scales: $0.5 \mathrm{~mm} \mathrm{(25)}$ and $0.1 \mathrm{~mm}\left(26^{-28}\right)$.

Euryopis mustelina Simon 1889b, p. 251 (holotype: 우 from Yokohama, Japan, A. Mellottée leg., in MNHN; not examin ed)-Bösenberg \& Strand 1906, p. 137, pl. 5, fig. 2, pl. 12, fig. 276; Saito 1941, p. 177, fig. 302; Yaginuma 1960, p. 34, pl. 7 , fig. 42.

Dipoena mustelina: Yaginuma 1967, p. 88, fig. 1a-c; Yaginuma 1968 , p. 34, pl. 7, fig. 42; Yaginuma 1986, p. 42, pl. 10, fig. 3, text-fig. 23-3; Chikuni 1989, p. 36, fig. 32; Zhu 1998, p. 240, fig. 157; Song et al. 1999, p. 112, fig. 55E-F, M; Yoshida \& Ono 2000, p. 135, figs. 17.

Distribution. Japan: Hokkaido, Honshu, Shikoku, Kyushu and the Nansei Islands. Korea, China and Indonesia.

\section{Trigonobothrys nigromaculatus (Yoshida 1987) new combination}

[Japanese name: Kurohoshi-mijingumo]

Pholcomma nigromaculatum Yoshida 1987, p. 29, figs. 1-4 (holotype: 우 from Shuishe, Nantow County, Taiwan, 29-III1979, H. Yoshida leg., NSMT-Ar 1871; examined).

Dipoena nigromaculata: Zhu 1998, p. 227, fig. 147; Song et al. 1999, p. 112, fig. 55G-H, N; Yoshida \& Ono 2000, p. 134.

Additional records. OKAYAMA: 1우소, Kamogawa-cho, 4V-1997, K. Nojima leg. SHIMANE: 1우, Iwatsubo, Izumo-shi,
25-VII-2001, Y. Ihara leg.; 1우, Kumura-Nenbutsuden, Izumoshi, 27-VII-2001, Y. Ihara leg.

Distribution. Japan: Honshu (Okayama and Shimane Prefectures) and the Nansei Islands (Iriomote Is.). China (Taiwan).

Euryopis Menge 1868 s. str.

[Japanese name: Hiratahimegumo zoku]

Euryopis Menge 1868, p. 174.

Euryopis s. lat.: Simon 1894, p. 528; Wiehle 1937, p. 131; Locket \& Millidge 1953, p. 44; Levi 1954, p. 3; Saito 1941, p. 177; Archer 1950, p. 13; Yaginuma 1960, p. 34; Levi \& Levi 1962, p. 39; Levi 1963, p. 126; Yaginuma 1986, p. 44; Wunderlich 1987, p. 209; Zhu 1998, p. 33.

Description. Carapace oval, not so high (Fig. 29). Abdomen flattened and dark color, usually with pale and silver flecks (Fig. 29). Median apophysis of male palpus absent; tegular apophysis attached to tegulum; cymbium with distal projection (Fig. 30). Epigynum usually with a depression. Colulus absent.

Notes. Euryopis iharai Yoshida 1992 is excluded in this paper, because the characteristics of this species are different from those of this genus. To which genus "Euryopis" iharai should be designated is uncertain. A generic revision of it will be made elsewhere in the near future. 

1836.

Type species. Micryphantes flavomaculata C. L. Koch

Euryopis flavomaculata (C. L. Koch 1836)

[Japanese name: Kimadara-hiratahimegumo]

(Figs. 29-30)

Micryphantes flavomaculata C. L. Koch 1836, p. 67, pl. 95, fig. 220 (holotype: ㅇ from Regensburg, Germany; not examined).

Euryopis flavomaculata: Wiehle 1937, p. 132, figs. 14-19; Locket \& Millidge 1953, p. 44, fig. 30; Levi 1954, p. 7, figs. 1, 8-10; Roberts 1985 , p. 174, pl. 104, text-fig. $77 \mathrm{~d}$; Yaginuma 1986, p. 44; Zhu 1998, p. 39, fig. 18; Song et al. 1999, p. 123, fig. 63E-F.

Additional records. HOKKAIDO: 1ㅅ, north shore of Nagafushi Lake, Toyokoro-cho, 27-VI-11-VII-1993, K. Shibata leg. (CMM); 1个, east shore of Nagafushi Lake, 11-24-VII-1993, K. Shibata leg. (CMM).

Distribution. Japan: Hokkaido and Honshu. Europe, Russia and China.
Euryopis octomaculata (Paik 1995)

[Japanese name: Yahoshi-hiratahimegumo]

Steatoda octomaculata Paik 1995 a, p. 7, figs. 8-13 (holotype: ㅇ from Nari-dong, Ulleung-do Island, Kyungpook, Korea, 26VII-1986, J. M. Kang leg., in KUT; not examined).

Euryopis octomaculata: Yoshida 1997, p. 111, figs. 1-5.

Distribution. Japan: Honshu (Okayama Pref.). Korea (Ulleung-do Is.).

Euryopis nigra Yoshida 2000

[Japanese name: Kuro-hiratahimegumo]

Euryopis nigra Yoshida 2000, p. 133, figs. 1-5 (holotype: $\hat{\jmath}$ from Kawabuchi, Shingu Dam, Shingu-mura, Uma-gun, Ehime Prefecture, Japan, 2-VIII-1994, Y. Ihara leg., NSMTAr 4520; examined).

Distribution. Japan: Honshu (Hiroshima Pref.), Shikoku (Ehime Pref.) and the Nansei Islands (Amami-oshima Is.).
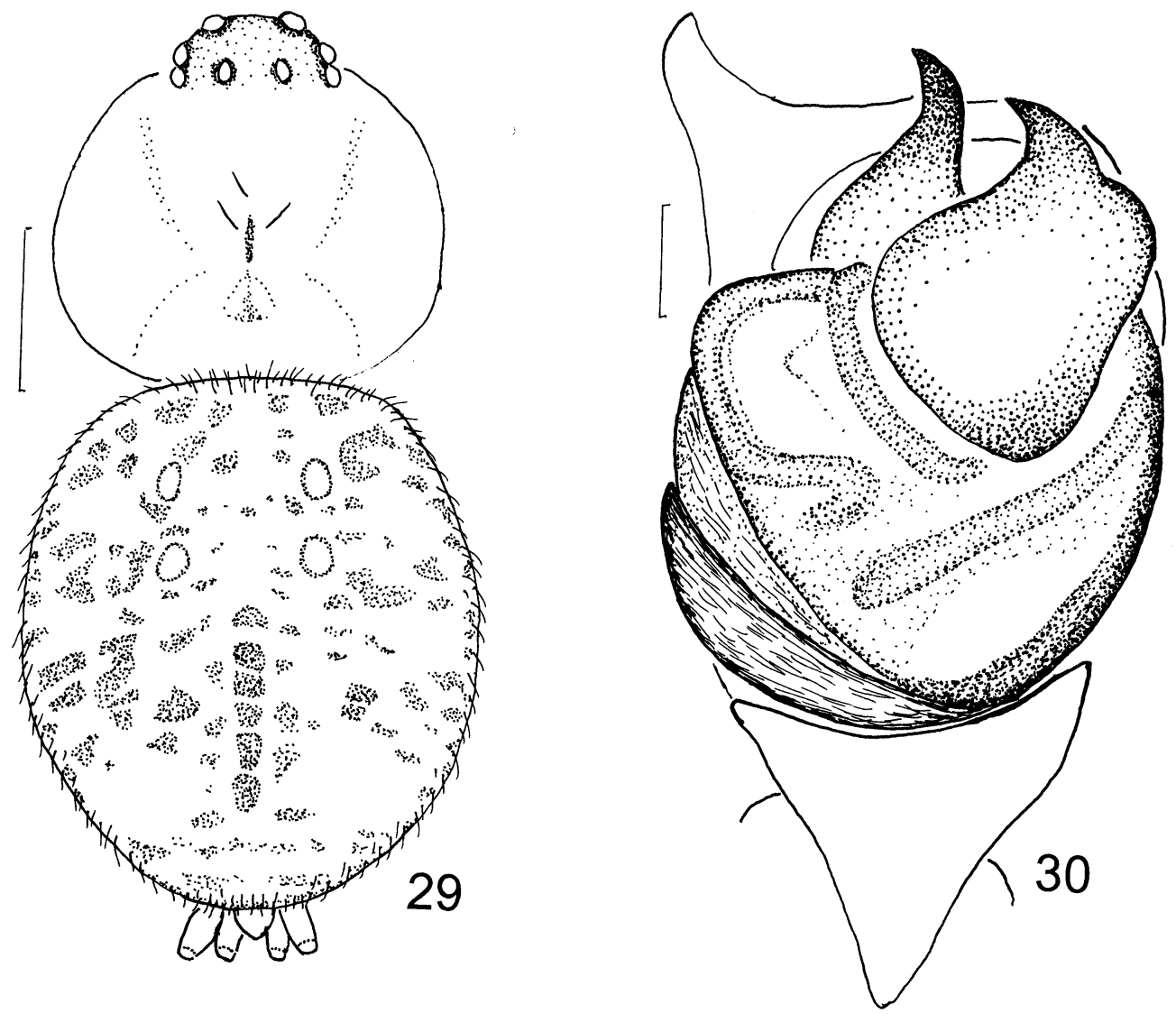

Figs. 29-30. Euryopis flavomaculata (C. L. Koch 1836), 令 from Toyokoro-cho, Hokkaido-29, carapace and abdomen, dorsal view; 30, left palpus, ventral view. Scales: $0.5 \mathrm{~mm} \mathrm{(29)}$ and $0.1 \mathrm{~mm}(30)$. 


\section{Emertonella Bryant 1949}

[Japanese name: Shirokane-hiratahimegumo zoku]

Emertonella Bryant 1949, p. 182.

Diagnosis. This genus resembles Euryopis, but is distinguished from it by carapace with high head region and cymbium of male palpus without distal projection.

Description. Head region of carapace high (Fig. 31). Abdomen triangular overhanging carapace (Fig. 31). Coloration of abdomen variable, usually black and silver. Epigynum with a large depression; seminal receptacles two pairs (Figs. 32-33). Cymbium of male palpus without projection (Fig. 34). Colulus absent.

Notes. Bryant (1949) had established this genus, but Levi (1954) synonymized it with Euryopis. Emertonella has been treated as the species group of E. emertoni, but I resurrect it as a genus in this paper. All the species of the E. emertoni group designed by Levi (1954) belong to this genus.

Type species. Euryopis emertoni Bryant 1933.

\section{Emertonella taczanowskii (Keyserling 1886) new combination}

[Japanese name: Shirokane-hiratahimegumo]

(Figs. 31-34)

Euryopis taczanowskii Keyserling 1886, p. 47, pl. 12, fig. 160 (holotype: ㅇ from Tumbes, Peru, in PAS; not examined)Levi 1963, p. 132, fig. 10; Levi 1967, p. 178, figs 37-41; Yoshida 1992, p. 139, figs. 1-4; Zhu 1998, p. 37, fig. 17;
Song et al. 1999, p. 123, fig. 63C-D, G.

Euryopis nigripes Banks 1929, p. 86 (holotype: 우 from Mt. Hope, Panama Canal Zone, in MCZ; not examined)-Levi 1954 , p. 24 , figs. $38-52$.

Additional records. OKINAWA: $1 \hat{\circ}$, Tamagusuku Castle,

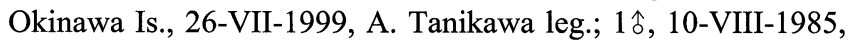

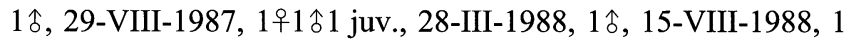
今, 29-III-1989, Urauchi, Iriomote Is., A. Tanikawa leg.

Distribution. Japan: Nansei Islands (Yoron Is., Izena Is., Okinawa Is. and Iriomote Is.). Widely distributed in the tropical area of Asia and America.

\section{Acknowledgments}

I wish to express my sincere thanks to Dr. Tsutomu Hikida, Kyoto University, Kyoto, Prof. Nobuo Tsurusaki, Tottori University, Tottori, and Dr. Hirotsugu Ono, National Science Museum, Tokyo, for their kind advice and to Dr. Akio Tanikawa, Kanagawa, for offering many specimens used in this paper. My thanks are also due to Ms. Mayumi Matsuda, Hokkaido, Mr. Yoh Ihara, Hiroshima, Mr. Koich Nojima, Osaka, Mr. Kiyoto Ogata, Aichi, Prof. Yoshiaki Nishikawa and Prof. Takahide Kamura, Otemon Gakuin University, Osaka, Prof. Hozumi Tanaka, Sonoda Gakuen Women's Junior College, Hyogo, and Mr. Nobuki Yasuda, Hokkaido, for offering or loaning the specimens.

\section{References}

Archer, A. F. 1950. A study of theridiid and mimetid spiders with descriptions of new genera and species. Alab. Mus. Nat. Hist., Mus. Pap., 30: 1-40.

Baert, L. 1984a. Mysmenidae and Hadrotarsidae from the Neotropical Guarani zoogeographical province (Paraguay and south Brasil) (Araneae). Rev. Suisse Zool., 91: 603-616.

Baert, L. 1984b. Spiders (Araneae) from Papua New Guinea. IV.
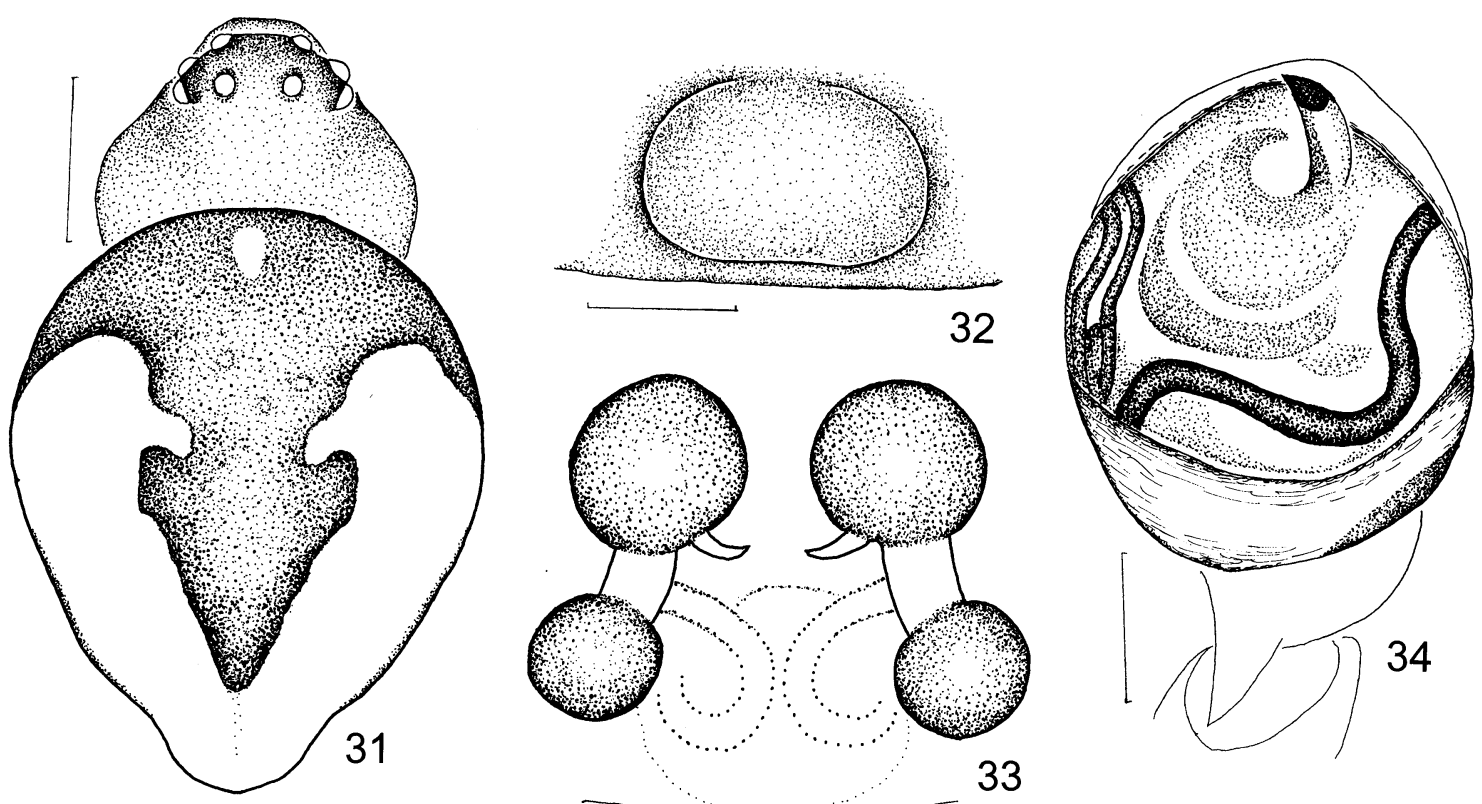

33

Figs. 31-34. Emertonella taczanowskii (Keyserling 1886), ㅇ from Okinawa Is., and 占 from Izena Is., Okinawa Pref.-31, female carapace and abdomen, dorsal view; 32, epigynum, ventral view; 33, female internal genitalia, dorsal view; 34, male left palpus, ventral view. Scales: $0.5 \mathrm{~mm}(31)$ and $0.1 \mathrm{~mm}(32-34)$. 
Ochyroceratidae, Telemidae, Hadrotarsidae and Mysmenidae. IndoMalayan Zool., 1: 225-244.

Banks, N. 1929. Spiders from Panama. Bull. Mus. Comp. Zool., 69: 53-96, pls. 1-2. (not seen)

Barrion, A. T. \& Litsinger, J. A. 1995. Riceland Spiders of South and Southeast Asia. CAB International, Wallingford. xix +700 pp., 16 pls.

Bösenberg, W. \& Strand, E. 1906. Japanische Spinnen. Abh. Senck. Naturf. Ges., 30: 93-422, pls. 3-10.

Bryant, E. B. 1949. A new genus and species of Theridiidae. Psyche, 56: 66-69. (not seen)

Chamberlin, R. V. 1948. On some American spiders of the family Erigonidae. Ann. Ent. Soc. America, 41: 483-562. (not seen)

Chen, J., Peng, J. \& Zhao, J. 1992. Two new species of theridiid spiders from China (Araneae: Theridiidae). J. Hubei Univ., Nat. Hist., 14: 270-274. (In Chinese with English summary.)

Chikuni, Y. 1989. Pictorial Encyclopedia of Spiders in Japan. Kaiseisha, Tokyo. 310 pp. (In Japanese)

Forster, R. R., Platnick, N. I. \& Coddington, A. J. 1990. A proposal and review of the spider family Synotaxidae (Araneae, Araneoidea) with notes on theridiid interrelationships. Bull. Amer. Mus. Nat. Hist., 193: 1-116.

Hickman, V. V. 1942. On some new Hadrotarsidae (Araneae) with notes on their internal anatomy. Pap. Proc. Roy. Soc. Tasmania, 147-169.

Ivie, W. 1967. Some synonyms in American spiders. J. New York Ent. Soc., 75: 126-131. (not seen)

Keyserling, E. 1886. Die Spinnen Amerikas. Theridiidae, part 2. Nürnberg 1886, 2 (2): 1-295, pls. 1-11.

Koch, C. L. 1836. Die Arachniden. III. Nürnberg 1836: 1-120, pls. 73-108.

Levi, H. W. 1953. Spiders of the genus Dipoena from America north of Mexico (Araneae, Theridiidae). Amer. Mus. Novitates, 1647: 139.

Levi, H. W. 1954. Spiders of the genus Euryopis from North and Central America (Araneae, Theridiidae). Amer. Mus. Novitates, 1666: 1-48.

Levi, H. W. 1963. American spiders of the genera Audifia, Euryopis and Dipoena (Araneae, Theridiidae). Bull. Mus. Comp. Zool., 129: 121-185.

Levi, H. W. 1967. Cosmopolitan and pantropical species of theridiid spiders (Araneae: Theridiidae). Pacific Insects, 9: 175-186.

Levi, H. W. \& Levi, L. R. 1962. The genera of the spider family Theridiidae. Bull. Mus. Comp. Zool., 127: 3-71, 14 pls.

Locket, G. H. \& Millidge, A. F. 1953. British Spiders II. Ray Society, London. vii +449 pp.

Menge, A. 1868. Preussische Spinnen. II. Abtheilung. Schr. Naturf. Ges. Danzig, (N. F.), 2: 153-218, pls. 28-43. (not seen)

Ohno, M. \& Yaginuma, T. 1968. The spiders from the Islands of Niijima, Shikinejima and Kozushima, belonging to the Izu Islands, Japan. J. Toyo Univ., Gen. Educ. (Nat. Hist.), 10: 17-29. (In Japanese with English summary)

Paik, K.-Y. 1995 a. Korean spiders of the genus Steatoda (Araneae: Theridiidae). I. Korean Arachnol., 11 (1): 1-14.

Paik, K.-Y. 1995 b. Korean spiders of the genus Dipoena (Araneae: Theridiidae). I. Korean Arachnol., 11 (1): 29-38.

Paik, K.-Y. 1996. Korean spiders of the genus Dipoena (Araneae: Theridiidae). II. Description of two new species. Korean Arachnol., 12 (2): 41-46.

Petrunkevitch, A. 1928. Systema Araneorum. Trans. Connect. Acad. Arts Sci., 26: 1-270.

Platnick, N. I. 2001. The world spider catalog, version 2.0. American Museum of Natural History, online at http://research.amnh.org/ entomology/spiders/catalog 81-87/index html

Roberts, M. J. 1983. Spiders of the families Theridiidae, Tetragnathidae and Araneidae (Arachnida: Araneae) from Aldabra atoll. Zool. J. Linn. Soc., 77: 217-291.
Roberts, M. J. 1985. The Spiders of Great Britain and Ireland I \& III. Harley Books, Colchester. 229 pp. \& 256 pp.

Saito, S. 1941. Fauna Nipponica Vol. IX, Fas. II, No. II, Suborder Arachnomorphae, Tertasticta Trionycha I, Class Arachnoidea, Order Araneina. Sanseido, Tokyo. 220 pp. (In Japanese)

Simon, E. 1881. Les Arachnides de France, 5: 1-179.

Simon, E. 1889a. Etudes arachnologiques. $21^{\mathrm{e}}$ Mémoire. XXXI. Descriptions d'espèces et de generes nouveaux de Madagascar et de Mayotte. Ann. Soc. Ent. France (6), 8: 223-236. (not seen)

Simon, E. 1889b. Etudes arachnologiques. $21^{\mathrm{e}}$ Mémoire: XXXIII. Descriptions de quelques espèces recueilles au Japon, par A. Mellotèe. Ann. Soc. Ent. France (6), 8: 248-252.

Simon, E. 1893. Histoire Naturelle des Araignées, 2nd ed., 1: 257488.

Simon, E. 1894. Histoire Naturelle des Araignées, 2nd ed., 1: 489760.

Song, D., Zhu, M. \& Chen, J. 1999. The Spiders of China. Hebei Science and Technology Publishing House, Hebei. 640 pp., 4 pls.

Tanikawa, A. 2000. A check list of Japanese spiders (ver. 2000). Kishidaia, 78: 79-142. (In Japanese)

Thorell, T. 1869. On European spider. Part I. Review of the European genera of spiders, preceded by some observations on zoological nomenclature. N. Act. Reg. Soc. Sci. Upsal. (3), 7: 1-242.

Thorell, T. 1881. Studi sui ragni Malesi e Papuani. Part III. Ann. Mus. Civ. Stor. Nat. Genova (2), 8: 1-720.

Tullgren, A. 1949. Bidrag till kännedomen om den sevenska spindelfauna. III. Theridiidae. Ent. Tidskr., 70: 33-64. (not seen)

Wiehle, H. 1937. Theridiidae oder Haubennetzspinnen (Kugelspinnen). Tiewelt Dtsch., 33: 119-222.

Wunderlich, J. 1978. Zu Taxonomie und Synonymie der Taxa Hadrotarsidae, Lucarachne Bryant 1940 und Flegia C. L. Koch 1854, (Arachnida: Araneida: Theridiidae). Zool. Beit., 24: 25-31.

Wunderlich, J. 1987. Die Spinnen der Kanarischen Inseln und Madeiras. Triops Verlag, Langen. 435 pp.

Wunderlich, J. 1988. Die Fossilen Spinnen im Dominikanischen Bernstein. Beit. Araneol., 2: 1-378.

Yaginuma, T. 1960. Spiders of Japan in Colour. Hoikusha, Osaka. vi $+186+8$ pp., 56 pls. (In Japanese)

Yaginuma, T. 1967. Revision and new addition to fauna of Japanese spiders, with descriptions of seven new species. Let. Dept. Rev., Otemon Gakuin Univ., 1: 87-107. (In Japanese with English description)

Yaginuma, T. 1968. Spiders of Japan in Colour (revised and enlarged ed.). Hoikusha, Osaka. vi +197 pp., 56 pls. (In Japanese)

Yaginuma, T. 1986. Spiders of Japan in Color (new ed.). Hoikusha, Osaka. xxiv +305 pp., 64 pls. (In Japanese)

Yoshida, H. 1985. Three new species of the spider genera Phoroncidia and Pholcomma (Araneae, Theridiidae) from Japan. Proc. Jap. Soc. Syst. Zool., 31: 7-13

Yoshida, H. 1991. Two species of the genus Dipoena (Araneae: Theridiidae) from Japan. Acta Arachnol., 40: 33-35. (In Japanese with English summary)

Yoshida, H. 1992. Two species of the genus Euryopis (Araneae: Theridiidae) from Japan. Acta Arachnol., 41: 139-142.

Yoshida, H. 1997. A newly recorded species of the genus Euryopis (Araneae: Theridiidae) from Japan. Acta Arachnol., 46: 111-113.

Yoshida, H. 2000. A new species of the genus Euryopis (Araneae: Therididae) from Japan. Acta Arachnol., 49: 133-136.

Yoshida, H. \& Ono, H. 2000. The spiders of the genus Dipoena (Araneae: Theridiidae) from Japan. Bull. Natn. Sci. Mus., Tokyo (A), 26: 125-158.

Zhu, M. 1998. Fauna Sinica, Arachnida, Araneae, Theridiidae. Science Press, Beijing. ix +436 pp., 1 pl. (In Chinese with English summary)

Received December 4, 2001 / Accepted January 24, 2002 


\section{Acta Arachnologica Vol. 51, No. 1 掲載論文の和文要旨}

アシナガグモの生活史 (pp. 1-4)

吉田 真（干525-8577 滋賀県草津市野路東 1-1-1 立命館大 学理工学部生物工学科)

野外調查と飼育によって，アシナガグモ（Tetragnatha praedonia) の生活史を調べた。野外では, 新たに孵化したと思 われる子グモが，6月から9月の間に繰り返し出現した。出の う直後から育てられた 10 頭のうち， 5 頭（オス 2 頭とメス 3 頭）が成体となった，出のうから最終脱皮までの期間は，オス では 57-59日，メスでは 47-51 日であった。新たに孵化した子 グモの度重なる出現と短い生活史は, アシナガグモが年 2-3 世 代の回転率をもつことを示唆している.

\section{アカクモヒメバチによるサツマノミダマシへの寄生の初記録}

【短報】(pp. 5-6)

桝元敏也 ${ }^{1}$, 清水 勇 $^{1}$, 小西和彦 ${ }^{2}{ }^{1}$ 广 $522-2113$ 大津市上田上 平野町字大塚 509-3 京都大学生態学研究センター ; ${ }^{2}$ T 0628555 札幌市豊平区羊ヶ丘 1 独立行政法人 農業技術研究機 構 北海道農業研究センター虫害研究室)

アカクモヒメバチ Eriostethus rufus (Uchida, 1932) は従来 Araneus 属のクモに寄生するとされていた。しかし, 我々はこ の八チが別属である Neoscona 属のクモに寄生することを発見 した。アアカクモヒメバチとその宿主であるクモとの関係は再検 討する必要がある.

日本産ミジングモ亜科（クモ目：ヒメグモ科）の属および種の 検討 (pp. 7-18)

吉田 哉（テ990-2484 山形市篭田 2 丁目 7 番 16 号）

日本産のミジングモ亜科 Hadrotarsinae Thorell 1881 の属およ び種の検討をおこなった。この亜科の特徴は, 雌の受精のうが 2 対で雌の触肢の爪が背腹方向に扁平, 第 1 歩脚跗節腹面に特 化した毛があること捛よび糸疣前疮の中央に洗濯板状の突出部 があることである.

すべての種に検討を加え, 属の検索表を表し, 日本から 6 属 19 種を記録した。このうち, ヤギヌマミジングモ属（新称） Yaginumena を新属として記載し, ッッミジングモ属（新称） Trigonobothrys Simon 1889 およびシロカネヒラタヒメグモ属 （新称）Emertonella Bryant 1949 を属として復活した.さらに, アイチミジングモ属（新称）Lasaeola Simon 1881 に属する種 を日本より記録した。 これらの属に属する 12 種, ボカシミジ ングモ Yaginumena castrata (Bösenberg \& Strand 1906), コア カクロミジングモ Y. mutilata (Bösenberg \& Strand 1906), マダ ラミジングモ Y. maculosa (Yoshida \& Ono 2000), オキナワミ ジングモ Lasaeloa okinawana (Yoshida \& Ono 2000), ヨシダミ ジングモ L. yoshidai (Ono 1991)， ヨナミジングモL. yona (Yoshida \& Ono 2000), ヤマトミジングモ Trigonobothrys japonicus (Yoshida 1985), アマミミジングモ T. amamiensis
(Yoshida 1985), ホシミジングモ T. martinae (Roberts 1983), キベリミジングモ T. flavomarginatus (Bösenberg \& Strand 1906), カニミジングモ T. mustelinus（Simon 1889）およびクロホシミ ジングモ T. nigromaculatus (Yoshida 1987) はミジングモ属 Dipoena Thorell 1869 より, さらに 1 種, シロカネヒラタヒメ グモ Emertonella taczanowskii（Keyserling 1886) はヒラタヒメ グモ属 Euryopis Menge 1868 より新たに属を移動した。また, タニカワミジングモ (新称) Dipoena nipponica を新種として記 載した。さらに, 中国で記載された Dipoena immaculata Zhu 1998 をキべリミジングモ T. flavomarginatus の，また北アメリ 力産の種をタイプ種とする属 Pselothorax Chamberlin 1948 をア イチミジングモ属 Lasaeola の新参異名とした. フタホシヒラ タヒメグモ Euryopis iharai Yoshida 1992 は所属が不明確のため 本稿では除外した.

\section{近畿地方でナス属を食害するナミハダニ属（ハダニ科）の 1 新 種 (pp. 19-22)}

江原昭三 ${ }^{1}$, 大橋和典 ${ }^{2}{ }^{1}$ ₹ $680-0001$ 鳥取市浜坂 2 丁目 15-7 ; ${ }^{2} \mathbf{T} 606-8502$ 京都市左京区北白川追分町 京都大学大学院農学 研究科)

大阪・京都両市の市街地でナス属 (Solanum) の 3 種の植物 （イヌホオズキ，ワルナスビおよびナス）に多発していた八ダ 二を新種と認め, Tetranychus takafujii（ミッユビナミハダニ, 新称）と命名・記載した（ホロタイプは大阪市福島区淀川河川 公園のイヌホオズキから採集した令). 本種は, 国外の T. evansi Baker \& Pritchard に最むよく似ているが, 今の第 II 脚の爪間体 の形態で異なる. 挿入器は国外産の T. marianae McGregor のそ れにも似るが, 早っの脚の形態で相違する. 脚の毛の配列は日 本のアシノワハダニにも類似するが, 挿入器の形態で識別でき る.この八ダニは, 日本国内でナス属の主な害虫の一つになる 可能性を持っていると思われる.

\section{中国のアシダカグモ科 2. 長春コレクションに含まれる種}

Peter Jäger', Jiuchun $\mathrm{Gao}^{2}$, Rui $\mathrm{Fei}^{2}$ ('Institute für Zoologie, Johannes Gutenberg-Universität, Germany; ${ }^{2}$ Jilin University, P. R. China) (pp. 23-31)

アシダカグモ科の 2 新種 : Sinopoda angulata と S. fasciculata を記載した. Pseudopoda sp. cf. exiguoides と Pseudopoda 属の 種名未決定種をそれぞれを湖南省と四川省から記録した。アシ ダカグモ Heteropoda venatoria を広東省と雲南省から記録した。 ツユグモ Micrommata virescens を吉林省から初めて記録した。 Olios tiantongensis を同種とみられる2 雄をそれぞれ江蘇省と 湖南省から記録した。 O. menghaiensis を同種とみられる1早を 雲南省から記録した。 Eusparassus sanguinifrons Simon 1906 の 雌を初めて記載し, Olios へ転属した。 全種の生殖器を図示し た。(和訳：編集委員会) 